\title{
Wide area mapping of liquid crystal devices with passive and active command layers
}

\author{
Thomas Bennett ${ }^{1}$, Matthew Proctor ${ }^{2, \dagger}$, Jon Forster $^{1}$, Eleni Perivolari ${ }^{2}$, Nina Podoliak ${ }^{2}$, \\ Matthew Sugden ${ }^{2}$, Roger Kirke ${ }^{2}$, Thomas Regrettier ${ }^{3}$, Thomas Heiser $^{3}$, Malgosia \\ KACZMAREK ${ }^{2}$, AND Giampaolo D’Alessandro ${ }^{1, *}$ \\ ${ }^{1}$ Mathematical Sciences, University of Southampton, Southampton, UK \\ ${ }^{2}$ Physics and Astronomy, University of Southampton, Southampton, UK \\ ${ }^{\dagger}$ Current address: NKT Photonics, Unit 20 Compass Point, Ensign Way, Southampton SO31 4RA \\ *Corresponding author: dales@soton.ac.uk \\ ${ }^{3}$ Laboratoire ICUBE, Université de Strasbourg, CNRS, Strasbourg, FR
}

Compiled October 8, 2017

\begin{abstract}
We track the non-uniformity of a wide area liquid crystal device using multiple cross-polarised intensity measurements. They give us not only accurate estimates of the core physical liquid crystal parameters, such as elastic constants, but also spatial maps of the device properties, including the liquid crystal thickness and pretilt angle. A bootstrapping statistical analysis coupled with the multiple measurements gives us reliable error bars on all the measured parameters. (c) 2017 Optical Society of America
\end{abstract}

OCIS codes: 160.3710 Liquid crystals, 230.3720 Liquid-crystal devices, 230.1150 All-optical devices, 120.4290 Nondestructive testing, 120.4630 Optical inspection

http://dx.doi.org/10.1364/ao.XX.XXXXXX

\section{INTRODUCTION}

Liquid crystals are a material of choice in many optical devices [1]: displays [2], optical light modulators [3], liquid crystal light valves [4], diffractive waveplates [5] and diffraction elements [6], waveguides [7] soliton channels [8] and lenses [9], to name a few. In all these devices it is essential that the geometry of the cell and its liquid crystal-related properties, e.g. the pretilt angle, are uniform across a wide area. In the case of the display industry many steps are taken to ensure that this uniformity is reached, but in many smaller scale applications the only viable solution is to check and monitor the uniformity and occasionally discard device that do not satisfy appropriate quality requirements. It is therefore important to be able to check easily the wide area properties of a liquid crystal device and also to monitor its stability in time. This is a key issue also for more exotic and experimental liquid crystal devices that may include liquid crystals doped with particles [10-13] or active alignment layers that may, for example, be light sensitive [14-16]. In this cases, it is important not just to measure and monitor uniformity, but also to be able to do it in situ, with a minimally invasive procedure.

There are many techniques to measure some of these properties. For example, it is possible to obtain extremely accurate measurements of the gap thickness of an empty cell using interferometric methods in which the cell acts as a Fabry-Pérot cavity. However, this is bound to change during the filling process and during storage. While it is possible to measure variations in the liquid crystal thickness of a filled cell by simply shining white light through cross polarizers, this measurement is unable to separate the effect of thickness variation from other effects, e.g. pretilt angle, change of anchoring energy, deposition of surface charges. Similarly, it is possible to use the crystal rotation method [17] to measure the pretilt, but this procedure is rather cumbersome and it is non trivial to obtain accurate pretilt maps. Finally, it is possible to use fluorescent confocal polarizing microscopy to obtain reliable maps of the liquid crystal alignment throughout a cell $[18,19]$. This is an exquisite procedure, but not easy to use and requires specialized equipment and powerful computational methods.

This brief overview shows the importance of knowing the fundamental parameters of a liquid crystal, such as its elastic constants, when taking wide area measurements of a cell. For example, it is only using an accurate model that it is possible to separate the effect of pretilt and cell-gap thickness on the polarization of the light passing through a liquid crystal cell. Elastic constants have been measured and explored through different techniques, such as for example using the voltage induced Frederiks threshold in untwisted and twisted cells [20,21]. Another very commonly used method consists in measuring the crosspolarized intensity (CPI) of a monochromatic light beam passing through the cell as a function of the voltage applied to the 
cell.

Here we propose a simple method to obtain reliable maps of the device properties that is based on CPI measurements at multiple locations on the surface of the device. The method provides a snapshot of device optical quality and provides a quick and precise procedure to monitor its reliability. We have tested it with three planar cells filled with different nematic liquid crystals and different alignment layers, including photoconductive polymer layers. In all cases we have obtained reliable maps of the liquid crystal thickness and the pretilt angle, as well as the values of elastic constants. Moreover, by performing a boot-strapping statistical analysis coupled with multiple measurements we can obtain error bars on all the measured parameters. The technique presented here requires some prior information about the cell and the liquid crystal, for example the type of alignment (planar, homeotropic, twisted, etc), of liquid crystal (e.g. nematic, cholesteric) and its dielectric anisotropy (negative or positive) and birefringence. Its principles are, however, quite general and require "just" developing a new fitting model of the CPI. For example, in this paper we have used a FrankOseen model for the alignment of a nematic liquid crystal. A ferroelectric liquid crystal would require a different model, but other aspects of the methodology described here would remain unchanged. In particular, the statistical analysis discussed on the next page can be applied without any changes provided that the temperature of the samples can be maintained constant. Therefore, even though the cells considered here are all planar, there is no obstacle in principle to using different types of cells, e.g. twist. We will come back to this point in the conclusions.

This paper is organized as follows. In the next section we discuss the experimental setup and the procedure followed to measure the CPI at various locations on the surface of the planar cells. Section 3 starts with a description of the mathematical model of the liquid crystal alignment that we have used to fit the data. It then gives details of the fitting procedure and of the statistical analysis that we have performed on the experimental data to extract the device parameters and their error bars. The results of our analysis on three different planar liquid crystal cells is contained in section 4 , while the last section summarizes the work presented and discusses its significance and how it can be extended and improved further.

\section{EXPERIMENTAL SETUP}

In order to measure the thickness of a liquid crystal layer over a wide area, a CPI measurement is performed at different locations in the cell. The relevant set-up is shown in figure 1. A light beam emitted by a He-Ne laser with $632.8 \mathrm{~nm}$ wavelength passes a polariser oriented at $45^{\circ}$ to the director alignment and enters the liquid crystal cell at normal incidence. After propagation through the cell, it passes through a second polariser, at $90^{\circ}$ to the first, and hits a photodiode that measures its intensity. An AC voltage is applied to the cell to reorient the liquid crystal. The two polarization components of the incident light experience different phase delays when passing through the liquid crystal cell. As a result, the beam output polarization may change, causing the CPI to vary as a function of the liquid alignment and, hence, of the voltage applied to the cell.

The CPI as a function of voltage is measured by applying a $10 \mathrm{kHz}$ AC voltage to the liquid crystal cell, and ramping the voltage peak-to-peak amplitude from 0 to $20 \mathrm{~V}$ in incremental steps of $0.1 \mathrm{~V}$. At each voltage step, the experiment controlling software analyses any transient effects by monitoring CPI,

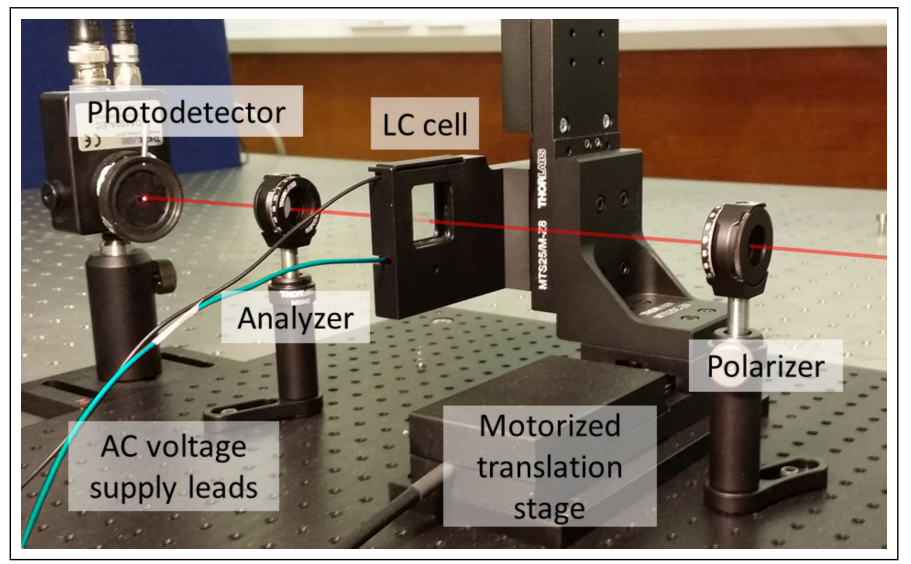

Fig. 1. Experimental set-up to measure the CPI at different locations of a planar liquid crystal (LC) cell. The polariser and analyzer are perpendicular to each other and at $45^{\circ}$ to the liquid crystal alignment. The motorized, computer controlled translation stage, allows us to define the area of the cell to be examined.

waits until the value saturates, and then takes and averages the intensity reading over $1 \mathrm{~s}$. The average CPI is recorded as a function of the rms amplitude of the applied voltage.

The cell is mounted on an assembly of two motorized translation stages from Thorlabs that move it with respect to the light beam in the plane of the cell, so that the same CPI measurement can be taken at different locations. Once the intensity has been measured for every voltage amplitude at one location on the cell, the cell is translated and the measurement is repeated at another location in order to map the liquid crystal thickness and pretilt across the whole cell area. As discussed in the Results section, it is important to keep the temperature constant for the duration of the experiment in order to have accurate estimates of the liquid crystal parameters. While we did not use a dedicated, temperature stabilized cell holder, we took some precautions to control the ambient temperature around the setup. Using just a simple holder allowed us to assess how critical it was to fix the temperature very precisely. We concluded that as only low laser power was required, it did not change the temperature of the sample during a single set of measurements in any considerable way. However, we note that there may have been variations in temperature between measurements for different samples.

The experiment is fully controlled by a specially developed software in Matlab, which perform experimental apparatus control, data acquisition and subsequent fit as described in the next sections. The software graphical user interface allows setting a range of $\mathrm{AC}$ voltages and coordinates on the cell surface where the measurements are taken.

This technique is designed to be applicable to any prototype liquid crystal device consisting of a planar liquid crystal layer of sufficient thickness to fulfill the half-wave retardation condition for the probe wavelength. This condition, while not essential, makes the measurement procedure more robust because of the large achievable variation in the light polarization as a function of the applied voltage. We tested it using two types of liquid crystal cells, all with anti-parallel planar alignment.

The first, marked as PI type, consisted of standard liquid crystal cells formed by two glass substrates coated with an ITO 
conductive layer and rubbed polyimide (PI) layer that produce uniform planar liquid crystal alignment. The substrates had size $2.5 \times 2.5 \mathrm{~cm}^{2}$ and were held together with UV-cured glue. The liquid crystal thickness was controlled by $12 \mu \mathrm{m}$ spacers deposited at the corners of the substrates. The cells were filled using capillary action and sealed with epoxy glue around the edges. We made two cells of this type using two different liquid crystals, namely E7 and TL205.

The second cell type, marked as P3HT, was similar to the first, except that it had a more complex hybrid layer structure consisting of two types of polymer mixtures: poly(3-hexylthiophene) and phenyl-C61-butyric acid methyl ester (P3HT:PCBM) with (1:1) weight ratio, and poly(3,4ethylenedioxythiophene) polystyrene sulfonate (PEDOT:PSS). The P3HT:PCBM layer is light sensitive [22], while PEDOT:PSS is a conducting polymer that can be used as liquid crystal alignment layer [23]. The liquid crystal layer was sandwiched between two ITO covered glass substrates, one coated with a $30 \mathrm{~nm}$ thick PEDOT:PSS layer and the other with a bi-layer structure of a $100 \mathrm{~nm}$ thick P3HT:PCBM and $90 \mathrm{~nm}$ thick PEDOT:PSS. The sample area was $1 \times 1 \mathrm{~cm}^{2}$ and the liquid crystal thickness was set by $7.75 \mu \mathrm{m}$ spacers. We made one cell of this type and filled with E7.

\section{DATA MODELING AND ANALYSIS}

\section{Modeling the cross-polarized intensity}

As we are working at high frequency and the voltage amplitude changes only gradually, we can neglect fluid motion in the liquid crystal and model its alignment using a FrankOseen theory[24, 25]. We identify the plane of the cell with the $(x, y)$-coordinate plane, with the entry facet at $z=0$. The director field $\hat{\boldsymbol{n}}$ is assumed to be uniform in $x$ and $y$ and is identified by the angle $\theta$ that it forms with the $x$-axis, $\hat{\boldsymbol{n}}=[\cos (\theta(z)), 0, \sin (\theta(z))]$. The governing equation takes the form [25]:

$$
\begin{aligned}
\gamma_{1} \frac{\partial \theta}{\partial t}= & {\left[K_{1} \cos ^{2}(\theta)+K_{3} \sin ^{2}(\theta)\right] \frac{\partial^{2} \theta}{\partial z^{2}} } \\
& +\frac{K_{3}-K_{1}}{2} \sin (2 \theta)\left(\frac{\partial \theta}{\partial z}\right)^{2}+\frac{\epsilon_{a} \epsilon_{0}}{2}\left(\frac{\partial \phi}{\partial z}\right)^{2} \sin (2 \theta) .
\end{aligned}
$$

The electric potential is given by

$$
\phi(z, t)=\int_{0}^{z} \frac{V(t)}{1+\frac{\epsilon_{a}}{\epsilon_{\perp}} \sin ^{2}(\theta)} d z^{\prime}\left(\int_{0}^{d} \frac{1}{1+\frac{\epsilon_{a}}{\epsilon_{\perp}} \sin ^{2}(\theta)} d z^{\prime}\right)^{-1}
$$

In these equations $\gamma_{1}$ is the liquid crystal rotational viscosity, $K_{1}$ and $K_{3}$ are the splay and bend elastic constants, $\epsilon_{0}$ is the vacuum permittivity, $\epsilon_{a}=\epsilon_{\|}-\epsilon_{\perp}$ the dielectric anisotropy of the nematic, with $\epsilon_{\|}$and $\epsilon_{\perp}$ the component of permittivity along and orthogonal to the director respectively, and $V(t)$ is the amplitude of the voltage applied to the liquid crystal layer. The boundary conditions on equation (1) are that the director forms a small angle with the cell sides, i.e. the orientation is approximately planar: $\theta(0) \simeq \theta(d)=\theta_{0}$, where $d$ is the liquid crystal thickness and $\theta_{0}$ is a small angle assumed to be approximately the same on the two cell sides. While it is easy in the model to relax this last assumption, in practice the CPI is insensitive to small differences in the pretilt angles on the two cell sides.

We define the CPI to be the ratio between the output and input intensity, so that it is normalized in the interval $[0,1]$. With

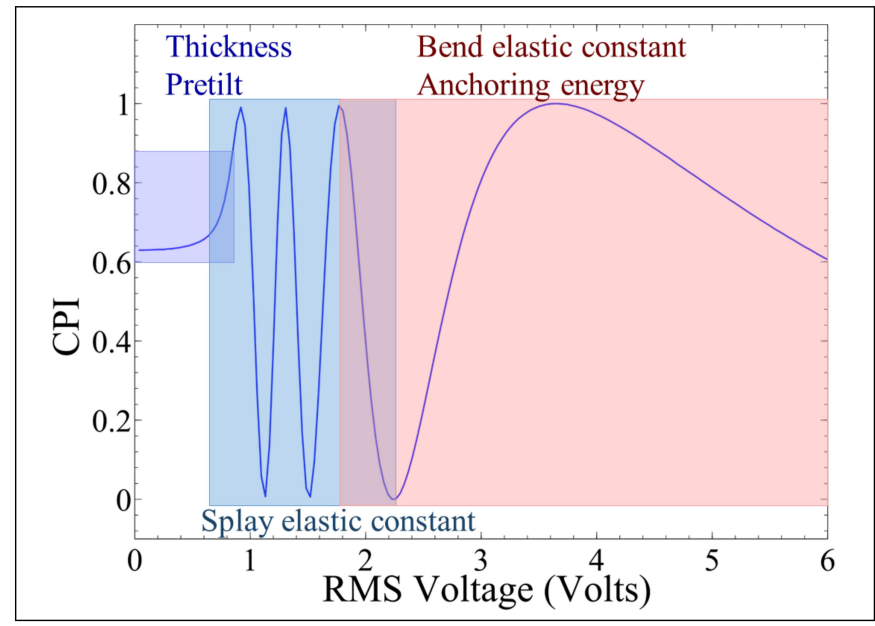

Fig. 2. Cross polarized intensity as a function of the applied voltage for an E7 cell. The shaded areas indicate which parts of the curve are more sensitive to the corresponding parameters in equation (1). The liquid crystal thickness is $\sim 11 \mu \mathrm{m}$.

a given solution for $\theta(z, t)$, the CPI, $I(t)$ is given by

$$
I(t)=\sin ^{2}\left(\frac{\pi}{\lambda} \int_{0}^{d}\left[n_{0}-n_{\mathrm{eff}}(\theta)\right] d z\right),
$$

where $n_{\text {eff }}$ is the effective refractive index seen by the component of polarization in the plane of the director,

$$
n_{\mathrm{eff}}(\theta)=\frac{n_{e} n_{0}}{\sqrt{n_{0}^{2} \cos ^{2}(\theta)+n_{e}^{2} \sin ^{2}(\theta(z))}} .
$$

Here $n_{o}$ and $n_{e}$ are the optical ordinary and extra-ordinary refractive indexes respectively.

\section{Statistical data analysis}

The statistical data analysis performed on the multiple traces is a generalization of the standard CPI trace analysis. For completeness and clarity we summarize this here, before discussing its generalization to multiple traces.

A typical CPI trace is shown in Figure 2. The region below the Frederiks transition is mainly sensitive to the liquid crystal thickness $d$ and the pretilt angle $\theta_{0}$. The splay elastic constant $K_{1}$ plays a role in the neighborhood of the Frederiks transition, while the bend elastic constant $K_{3}$ controls the high voltage part of the curve. We use the following fitting procedure for a single CPI trace. Firstly, the program detects all the minima and maxima and from their number obtains an approximate liquid crystal thickness. Then it fits the low voltage part of the curve (up to the first extremum) using the liquid crystal thickness and the pretilt angle as fitting parameters, while keeping the given values for elastic constants fixed. Refractive indices and dielectric constants of the liquid crystal are assumed known. These can be either taken from the liquid crystal data sheets or measured separately. After that, we fit the whole CPI trace taking the already found values for $d$ and $\theta_{0}$ and using $K_{1}$ and $K_{3}$ as fitting parameters.

The fitting procedure for multiple traces is an extension of the single trace algorithm. We use the following notation: we indicate with $x_{i}, i=1,2, \ldots, N_{p}$ the coordinates of the $N_{p}$ locations of the cell where the CPI has been measured. The applied 


\begin{tabular}{|l||c|c|}
\hline$N_{s}$ & $\hat{K}_{1}$ & $\hat{K}_{3}$ \\
\hline 2 & $10.70 \pm 0.016$ & $16.20 \pm 0.023$ \\
6 & $10.70 \pm 0.020$ & $16.20 \pm 0.037$ \\
12 & $10.66 \pm 0.022$ & $16.23 \pm 0.036$ \\
24 & $10.64 \pm 0.017$ & $16.25 \pm 0.028$ \\
\hline
\end{tabular}

Table 1. Average values of $K_{1}$ and $K_{3}$ (with estimated errors) for the PI-E7 cell using different number of subsets. Total number of sampling locations is $N_{p}=48$.

voltage takes $N_{V}$ values, $V=V_{j}, j=1,2, \ldots, N_{V}$, so that the result of an experiment is a set of $N_{p}$ traces, each consisting of $N_{V}$ values of the CPI.

We assume that the liquid crystal parameters, i.e. $K_{1}$ and $K_{3}$, have the same values at each sampling location $x_{i}$, while the surface parameters may be position dependent. We indicate the thickness and pretilt at $x_{i}$ with $d_{i}$ and $\theta_{0}^{(i)}$, respectively.

In order to keep the fitting procedure manageable, we bunch the $N_{p}$ experimental CPI traces in $N_{s}$ subsets, each with $N_{T}$ traces, so that $N_{p}=N_{s} \times N_{T}$. We fit all the traces in each subset with $N_{T}$ values of $d_{i}$ and $\theta_{0}^{(i)}$ (one pair per trace), but only one value of $K_{1}$ and $K_{3}$, using for each trace the single-trace procedure outlined at the start of this section. At the end of this step we have $N_{p}$ values of the thickness and pretilt, $d_{i}$ and $\theta_{0}^{(i)}$, one for each sampling location $x_{i}$, and $N_{s}$ values of $K_{1}$ and $K_{3}$, which we indicate with $K_{q}^{(m)}, m=1,2, \ldots, N_{s}, q=\{1,3\}$.

To estimate the mean value $\hat{K}_{q}$ and error $\Delta K_{q}$ of an elastic constant $K_{q}$ we simply compute the mean and standard deviation:

$$
\begin{aligned}
\hat{K}_{q} & =\frac{1}{N_{s}} \sum_{m}^{N_{s}} K_{q}^{(m)} \\
\Delta K_{q} & =\sqrt{\frac{1}{N_{s}-1} \sum_{m}^{N_{s}}\left[K_{q}^{(m)}-\hat{K}_{q}\right]^{2}} .
\end{aligned}
$$

These values are relatively insensitive to the number of subsets (see table 1).

We use parametric bootstrapping [26] to quantify the error in the estimation of $d$ and $\theta_{0}$ across the cell. We assume that the $N_{V}$ data measured at each site corresponds to sampling from an unknown multivariate normal distribution $\mathcal{N}(\boldsymbol{\mu}(\boldsymbol{x}), \Sigma)$ with mean vector $\boldsymbol{\mu}(\boldsymbol{x})$ and covariance matrix $\Sigma$. Here $\mu \in \mathbb{R}^{N_{V}}$ and $\Sigma$ is an $N_{V} \times N_{V}$ matrix. This notation emphasizes the fact that the CPI values at different voltages are not considered independent. We have allowed for the mean CPI, $\mu(x, V)$ to vary with position to account for variations in thickness, pretilt or other parameters. Conversely, sources of error, which are captured by the covariance matrix, are assumed to be identical at every location. Hence, the covariance matrix does not depend on position within the cell.

The measured CPI at position $x_{i}$ and voltage $V_{j}$ is indicated with $I_{\exp }\left(x_{i}, V_{j}\right)$ or with the more compact vector notation

$$
\boldsymbol{I}_{\exp }^{(i)}=\left[I_{\exp }\left(\boldsymbol{x}_{i}, V_{1}\right), \ldots, I_{\exp }\left(\boldsymbol{x}_{i}, V_{N_{V}}\right)\right]
$$

The corresponding theoretical CPI value, obtained from equa-
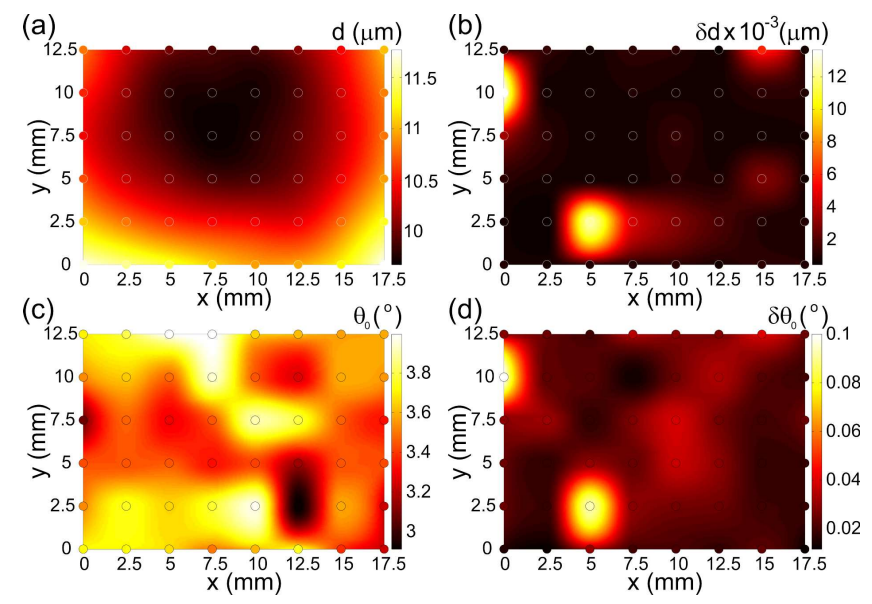

Fig. 3. PI planar cell filled with E7: Spatial map of the liquid crystal (a) thickness and (c) pretilt angle; (b) and (d) are the corresponding errors. Circles are the fitted values and the background color map is a piece-wise cubic interpolation between them. Fitting parameters: $N_{p}=48, N_{s}=12, N_{B}=8$.

tion (3), is indicated with

$$
\boldsymbol{I}_{\mathrm{th}}^{(i)}=\left[I\left(V_{1} ; \hat{K}_{1}, \hat{K}_{3}, d_{i}, \theta_{0}^{(i)}\right), \ldots, I\left(V_{N_{V}} ; \hat{K}_{1}, \hat{K}_{3}, d_{i}, \theta_{0}^{(i)}, V_{N_{V}}\right)\right] .
$$

The covariance matrix $\Sigma$ is estimated by [26]

$$
\hat{\Sigma}=\frac{1}{N_{p}-1} \sum_{i=1}^{N_{p}}\left[\boldsymbol{I}_{\exp }^{(i)}-\boldsymbol{I}_{\mathrm{th}}^{(i)}\right]\left[\boldsymbol{I}_{\exp }^{(i)}-\boldsymbol{I}_{\mathrm{th}}^{(i)}\right]^{T} .
$$

The bootstrapping procedure consists in using the multivariate normal distribution $\mathcal{N}\left(\boldsymbol{I}_{\mathrm{th}}^{(i)}, \hat{\Sigma}\right)$ to generate a number $N_{B}$ of CPI traces for each sampling location $x_{i}$. The low voltage part of each of them is fitted for the liquid crystal thickness and pretilt. In this way at each sampling location we obtain $N_{B}$ values of these two parameters; we use their standard deviation as the estimate of the error on $d_{i}$ and $\theta_{0}^{i}$. The value $N_{B}=8$, used in the analysis of our data, is perhaps smaller than ideal, but nonetheless seems to give a reasonable estimate of the error, especially in light of the magnitude of the systematic errors, as we discuss in the next section.

\section{RESULTS}

We have followed the procedure outlined above to measure the splay and bend elastic constants of E7 and TL205 in two PI and one P3HT cells. We have also measured the thickness and pretilt across the surfaces of the three cells and their corresponding errors. The He-Ne laser used in these measurements is outside the photo-activation region of the P3HT:PCBM so that this polymer can be considered as an inert dielectric from the point of view of these measurements. We present here graphical data only for the PI-E7 and P3HT-E7 cells. The data for the PI-TL205 cell is very similar.

Typical thickness maps are shown in Figure 3(a) and Figure 4(a) for a PI-E7 and a P3HT-E7 cell respectively. In both cases the thickness is smaller in the center of the cell, probably due to the squeezing of the cell when sealing it. The fact that the liquid crystal thickness in the P3HT-E7 cell varies by $3 \mu \mathrm{m}$ highlights the importance of keeping the pressure on the cell in 

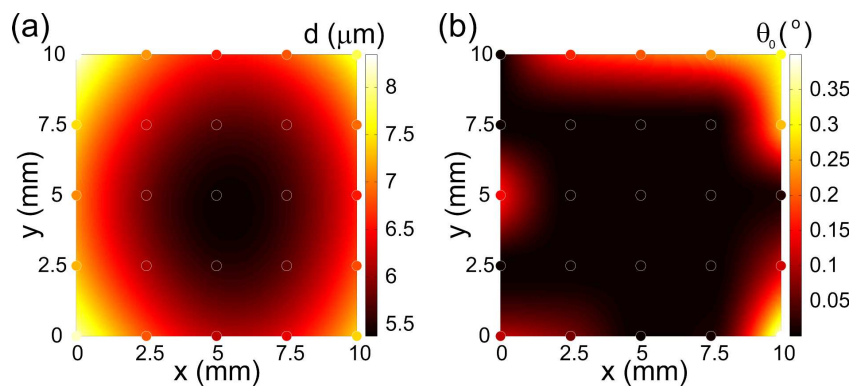

Fig. 4. P3HT cell filled with E7: Spatial map of the liquid crystal (a) thickness and (b) pretilt angle. Circles are the fitted values and the background color map is a piece-wise cubic interpolation between them. Fitting parameters: $N_{p}=25, N_{S}=5$.

the last stages of assembly as small and as uniform as possible. The PI-TL205 cell had a profile very similar to the PI-E7 cell.

Figure 3(c) presents a typical example of a liquid crystal cell with a pretilt of approximately $3^{\circ}$. At first sight, the pretilt maps look less structured than the thickness maps. However, it is important to notice that the angle variation is very small. In the case of the PI-E7 cell the variation is less than $1^{\circ}$. The pretilt in the P3HT-E7 cell is virtually $0^{\circ}$ everywhere except close to the boundaries of the cell, where imperfections due to the gluing process may affect the liquid crystal alignment. A similar behavior is also displayed by the PI-TL205 cell: the pretilt near the center of the cell varies between $1^{\circ}$ and $2^{\circ}$, with more significant variations only close to the boundaries.

The error maps, shown only for the PI-E7 cell (the other cells have very similar maps), figures $3(b, d)$, indicate that the estimation error, both on the thickness, $\delta d$, and on the pretilt angle $\delta \theta_{0}$, are in general small and uniform: the thickness and pretilt variations are of the order of a few nanometers and a few tenths of degrees respectively. The only glaring exception is the location at coordinates $(5,2.5) \mathrm{mm}$. The much larger error at this location is not due to an imperfection in the cell (which is clearly absent in the plots on the left hand side of this figure). It is caused, instead, by the limitation of fitting the CPI. In the CPI trace shown in figure 2 the value at $V=0$ is well away from 0 and 1: a small perturbation of the cell or liquid crystal parameters (as in the bootstrapping procedure) will not qualitatively change this graph. However, in the case of the location at coordinates $(5,2.5) \mathrm{mm}$ in figure $3(\mathrm{~b}, \mathrm{~d})$, the $\mathrm{CPI}$ at $V=0$ is very close to 1. A small perturbation can, in this case, create or remove a maximum from the CPI trace thus changing the graph qualitatively. This perturbation increases significantly the error in the estimate because, even though it remains small and does not affect conspicuously the mean, i.e. the thickness and pretilt plotted in figures $3(\mathrm{a}, \mathrm{c})$ respectively.

Finally, the estimates of the splay and bend elastic constants and their errors are listed in table 2 . We discuss here only the case of E7, for which there is more literature material. First we note that the values of the E7 elastic constants are compatible with other estimates that have appeared in the literature, summarized in table 3 . The second observation is that the error estimates are smaller than the differences in the values of $K_{1}$ and $K_{3}$ that we have ourselves measured or that have been reported in the literature. This is caused by the fact that there are many systematic errors that are not included in the measurement process. We discuss here four, as examples.

The first is temperature. Applying a standard error propa-

\begin{tabular}{llcc}
\hline LC & Cell type & $K_{1}[\mathrm{pN}]$ & $K_{3}[\mathrm{pN}]$ \\
\hline E7 & PI & $10.7 \pm 0.02$ & $16.2 \pm 0.03$ \\
E7 & P3HT & $10.9 \pm 0.04$ & $18.0 \pm 0.07$ \\
TL205 & PI & $17.0 \pm 0.05$ & $23.0 \pm 0.16$ \\
\hline
\end{tabular}

Table 2. Splay and bend elastic constants measured for the two PI cells and one P3HT cell analyzed in this paper. Fitting parameters are $N_{p}=48, N_{s}=12$ for the PI cells and $N_{p}=25$, $N_{S}=5$ for the P3HT cell. See text for interpretation of the error bars, including the effect of temperature.

\begin{tabular}{lcc}
\hline Reference & $K_{1}[\mathrm{pN}]$ & $K_{3}[\mathrm{pN}]$ \\
\hline Raynes et al (1979) [27] & 11.7 & 19.5 \\
Raynes et al (2003) [21] & 11.2 & 18.6 \\
Trabi et al (2008) [28] & 10.5 & 15.2 \\
Chen et al (2015) [29] & 10.8 & 17.5 \\
\hline
\end{tabular}

Table 3. Some literature values for the E7 splay and bend elastic constants.

gation technique to the temperature dependence formulas [30] for $K_{1}$ and $K_{3}$ shows that a $1{ }^{\circ} \mathrm{C}$ temperature variation around room temperature induced a percentage error in $K_{1,3}$ of more than $1 \%$.

Another effect is that the glass slides of the cell form a FabryPérot cavity: changing the alignment of the liquid crystal moves the light in and out of resonance with the cavity and, hence, modulates the CPI. As the liquid crystal thickness is not uniform the cell sides are curved: they are nearly parallel in the cell center, but more at an angle near the cell boundaries. Hence the quality of the Fabry-Pérot cavity, and hence, the CPI modulation is position dependent.

The third effect to consider is more significant for the measurements of $K_{3}$. As illustrated in figure 2 this parameter is determined by the large voltage amplitude part of the CPI trace. For these voltage values most of the liquid crystal in the bulk is aligned with the electric field and the only significant alignment variations are near the boundaries. It is likely that surface effects, like surface anchoring energy, charge deposition, dust, affect these measurements more significantly than those of $K_{1}$, whose value is determined by the liquid crystal deflection in the bulk.

The fourth and last effect is the role that the thickness of the polymer layer plays on the voltage dropped across the liquid crystal layer. The voltage amplitude $V(t)$ that appears in equation Eq. (2) is the voltage applied to the liquid crystal layer. However, the value used in the parameter fitting is the voltage applied to the whole cell, because this is the only parameter we can measure. In general, the two voltages are very close one to the other, because the polymer layer is extremely thin with respect to the liquid crystal layer. For example, for the PI cells the two thicknesses are approximately $20 \mathrm{~nm}$ and $12 \mu \mathrm{m}$. However, this may not always be the case. For example, in the P3HT cells the equivalent of these two layers have thickness of $100 \mathrm{~nm}$ and $6 \mu \mathrm{m}$ respectively. This implies that we are slightly overestimating the voltage applied to the liquid crystal layer in the P3HT cells and, hence, also the magnitude of the elastic constants, as 
confirmed by the values reported in table 2 .

An uncharitable reader may at this juncture ask why bother with the error bars reported in table 2. The point we wish to make is that they are not an estimate of the error on the "true" value of the splay and bend elastic constant. They are, instead, a measurement of the reproducibility of the cell fabrication process, provided that the measurements are temperature controlled. If the fabrication process is such that the systematic errors are controlled, in the sense that they are the same for all cells, then we expect that the measured values of the elastic constants will fall within the estimated error bars.

\section{CONCLUSIONS}

We have developed a fully automated process to estimate the non-uniformity of wide-area liquid crystal cells and to measure liquid crystal parameters. The experimental procedure is relatively straightforward and allows minimal-disruption measurements, thus making it ideal to establish the long-time in situ stability of new compounds. The measurements are fully computer controlled and can be set up using a graphical user interface. The data obtained can be fitted in real time on a standard PC. A statistical analysis based on bootstrapping methods gives not only the mean values of the desired parameters, but also estimates of the error bars. These can be used to verify the uniformity of the cell fabrication process. Adding frequency control to the voltage signal generator enables the use of this set-up also to measure the liquid crystal viscosities, using the procedure described in [31]. In this way a single experiment can determine both the static and dynamic properties of a liquid crystal.

In this paper we have focused on planar cells with the same alignment layers on both substrates, so they have similar pretilt angles on both sides. The pretilt angles, varying between $0^{\circ}$ and $4^{\circ}$ had standard, typical values. However, our approach is not confined to such cases; neither the pretilt angle magnitudes nor the symmetry of the alignment layers impose an intrinsic restriction on this method. We determine the pretilt using the low voltage part of the CPI trace, i.e. the region near the Frederiks threshold. This is sensitive only to the average pretilt. However, the liquid crystal alignment as a function of voltage depends on the two separately. It is therefore possible, in principle, to measure different pretilts on the two cell sides. However, it is likely that the error bar on the difference will be large, so that the measurement is significant only for sufficiently different alignment layers. In the case of twist cells or planar-homeotropic cells the alignment and, hence, CPI dynamics are different from those considered here in the sense that the model in equations (1-4) will need changing, but neither the measurement process nor the fitting procedure and statistical data analysis will require alteration.

Finally, we are exploring the possibility of using this method to measure and analyze other surface properties, like, for example, the anchoring energy or the effect of surface charges. These effects are small and likely to be detectable only at high voltage, through changes in the measured values of the bend elastic constant $K_{3}$. This may require to control many aspects of the experiments, from the Fabry-Pérot effect mentioned in the previous section to the small relative twist between the two cell sides which normally has a negligible effect on the CPI. Moreover, it is possible that two or more parameters may be degenerate, in the sense that a change in one can be compensated by a change in the other. For example, an increase in birefringence can be compensated to first order by reducing the cell thickness. Lifting the degeneracy will require a careful analysis of the fitting model and possibly changes to the experimental control parameter region explored, e.g. the number of different applied voltage frequencies used. This type of measurements could be particular important for tracking the long-term stability of liquid crystal colloidal suspensions in which the surfactant required to disperse the particle may react with the alignment layers on a long-time scale.

This work has been partially supported by the Royal Society's Paul Instrument Fund (PI150041)) and DSTL (grant DSTLX1000087813R and 1000105958).

The authors would like to thank Prof Tim Sluckin for useful discussions.

\section{REFERENCES}

1. J. Beeckman, K. Neyts, and P. J. M. Vanbrabant, "Liquid-crystal photonic applications," Opt. Eng. 50, 081202 (2011).

2. M. Schadt, "Liquid crystal materials and liquid crystal displays," Ann, Rev. Mat. Scien. 27, 305-379 (1997).

3. J. De La Tocnaye, "Engineering liquid crystals for optimal uses in optical communication systems," Liq.Cryst. 31, 241-269 (2004).

4. S. Residori, "Patterns, fronts and structures in a Liquid-Crystal-LightValve with optical feedback," Phys. Rep. 416, $201-272$ (2005).

5. S. R. Nersisyan, N. Tabiryan, D. Mawet, and E. Serabyn, "Improving vector vortex waveplates for high-contrast coronagraphy," Opt.Exp. 21, $8205-8213$ (2013).

6. A. K. Srivastava, X. Wang, S. Q. Gong, D. Shen, Y. Q. Lu, V. G. Chigrinov, and H. S. Kwok, "Micro-patterned photo-aligned ferroelectric liquid crystal fresnel zone lens," Opt. Lett. 40, 1643-1646 (2015).

7. D. Donisi, R. Asquini, A. d'Alessandro, and G. Assanto, "Distributed feedback grating in liquid crystal waveguide: a novel approach," Opt. Express 17, 5251-5256 (2009).

8. M. Peccianti, A. Dyadyusha, M. Kaczmarek, and G. Assanto, "Tunable refraction and reflection of self-confined light beams," Nature Physics 2 , 737-742 (2006).

9. C. Cui, R. Bao, S. Yu, and M. Ye, "Polarizer-free imaging using reference image for liquid crystal lens," Optics Communications 342, $214-217$ (2015).

10. T. Hegmann, H. Qi, and V. Marx, "Nanoparticles in Liquid Crystals: Synthesis, Self-Assembly, Defect Formation and Potential Applications," J. Inorg. Organomet. Polym. Mater. 17, 483-508 (2007). 10.1007/s10904-007-9140-5.

11. D. F. Gardner, J. S. Evans, and I. I. Smalyukh, "Towards reconfigurable optical metamaterials: Colloidal nanoparticle self-assembly and self-alignment in liquid crystals," Mol. Cryst. Liq. Cryst. 545, 3/[1227]21/[1245] (2011).

12. A. Mertelj, D. Lisjak, M. Drofenik, and M. Čopič, "Ferromagnetism in suspensions of magnetic platelets in liquid crystal," Nature 504, 237241 (2013).

13. N. Podoliak, O. Buchnev, M. Herrington, E. Mavrona, M. Kaczmarek, A. Kanaras, E. Stratakis, J.-F. Blach, J.-F. Henninot, and M. Warenghem, "Elastic constants, viscosity and response time in nematic liquid crystals doped with ferroelectric nanoparticles," RSC Advances 4, 46068-46074 (2014).

14. D. Evans, G. Cook, and M. Saleh, "Recent advances in photorefractive two-beam coupling," Opt. Mat. 31, 1059 - 1060 (2009). Selected Papers of the Third International Workshop on "Photonics and electronic materials".

15. M. Herrington, K. Daly, O. Buchnev, G. D'Alessandro, and M. Kaczmarek, "AC-field-enhanced beam coupling in photorefractive, hybrid liquid crystals," EPL 95, 14003 (2011).

16. M. Proctor, J. Bateman, K. Daly, M. Herrington, O. Buchnev, N. Podoliak, G. D'Alessandro, and M. Kaczmarek, "Light-activated modulation and coupling in integrated polymer-liquid crystal systems," J. Opt. Soc. Am. B 31, 3144-3152 (2014). 
17. F. Nakano, M. Isogai, and M. Sato, "Simple method of determining liquid crystal tilt-bias angle," Jpn. J. Appl. Phys. 19, 2013 (1980).

18. G. Posnjak, S. Čopar, and I. Muševič, "Points, skyrmions and torons in chiral nematic droplets," Sci. Rep. 6 (2016).

19. U. Mur, S. Čopar, G. Posnjak, I. Muševič, M. Ravnik, and S. Žumer, "Ray optics simulations of polarised microscopy textures in chiral nematic droplets," Liquid Crystals pp. 1-9 (2016).

20. J. Stromer, E. P. Raynes, and C. V. Brown, "Study of elastic constant ratios in nematic liquid crystals," Appl. Phys. Lett 88, 0519151-3 (2006).

21. E. P. Raynes, C. V. Brown, and J. F. Stromer, "Method for the measurement of the $k_{22}$ nematic elastic constant," Appl. Phys. Lett. 82, 13-15 (2003).

22. C. Soci, I.-W. Hwang, D. Moses, Z. Zhu, D. Waller, R. Gaudiana, C. Brabec, and A. Heeger, "Photoconductivity of a low-bandgap conjugated polymer," Advanced Functional Materials 17, 632-636 (2007).

23. T.-R. Chou, S.-H. Chen, Y.-T. Chiang, Y.-T. Lin, and C.-Y. Chao, "Highly conductive pedot:pss films by post-treatment with dimethyl sulfoxide for ito-free liquid crystal display," J. Mater. Chem. C 3, 3760-3766 (2015).

24. F. C. Frank, "I. Liquid crystals. On the theory of liquid crystals," Discuss. Faraday Soc. 25, 19-28 (1958).

25. I. W. Stewart, The static and dynamic continuum theory of liquid crystals: a mathematical introduction (Taylor \& Francis, 2004).

26. T. A. Severini, Likelihood Methods in Statistics (Oxford University Press, 2000).

27. E. P. Raynes, R. J. A. Tough, and K. A. Davies, "Voltage dependence of the capacitance of a twisted nematic liquid crystal layer," Mol. Cryst. Liq. Cryst. 56, 63-68 (1979).

28. C. L. Trabi, C. V. Brown, A. A. T. Smith, and N. J. Mottram, "Interferometric method for determining the sum of the flexoelectric coefficients $\left(e_{1}+e_{3}\right)$ in an ionic nematic material," Appl. Phys. Lett. 92, 223509 (2008).

29. H. Chen, R. Zhu, J. Zhu, and S.-T. Wu, "A simple method to measure the twist elastic constant of a nematic liquid crystal," Liq. Cryst. 42, 1738-1742 (2015).

30. H. Wang, T. X. Wu, S. Gauza, J. R. Wu, and S.-T. Wu, "A method to estimate the Leslie coefficients of liquid crystals based on MBBA data," Liq. Crys. 33, 91-98 (2006).

31. T. Bennett, M. Proctor, M. Kaczmarek, and G. D'Alessandro, "Lifting degeneracy in nematic liquid crystal viscosities with a single optical measurement," J. Colloid Interface Sci. 497, 201-206 (2017). 